\title{
Modified WKB Description of Bound States for the Gaussian Confining Potential
}

\author{
A. V. Baran*, V. V. Kudryashov ${ }^{\dagger}$ \\ Institute of Physics, National Academy of Sciences of Belarus \\ *Corresponding Author: a.baran@dragon.bas-net.by
}

Copyright (C)2013 Horizon Research Publishing All rights reserved.

\begin{abstract}
The bound states energies and eigenfunctions are obtained within the framework of the variational modified WKB approach for the Gaussian confining potential. The accuracy of the results is shown to be fairly good.
\end{abstract}

Keywords modified WKB method, radial wave functions, Gaussian potential

\section{Introduction}

The Gaussian confining potential

$$
V(r)=-A \frac{\hbar^{2}}{2 m R^{2}} \exp \left(-r^{2} / R^{2}\right)
$$

was used originally for the description of elastic scattering of nuclei by nuclei [1]. Later this attractive potential was applied in semiconductor physics in order to simulate the electron behavior in quantum dots [2]. The bound state energies for the Gaussian potential have been calculated in various papers [3-7] in the framework of different approximation techniques. However, the approximate eigenfunctions were studied insufficiently.

In the present paper, we use a new modified WKB method $[8,9]$ to solve the radial Schrödinger equation

$$
-\frac{\hbar^{2}}{2 m} \frac{d^{2} \psi(r)}{d r^{2}}+\left(V(r)+\frac{\hbar^{2} l(l+1)}{2 m r^{2}}-E\right) \psi(r)=0 .
$$

One of the earliest and simplest methods of obtaining the approximate eigenvalues and eigenfunctions is the WKB method which was described in many books (see, e.g., $[10,11])$. In the context of WKB approach it is well known [11] that a suitable transformation of the initial equation improves the results of an approximation. We examine the power-law substitutions

$$
r=q^{s}, \quad s>0, \quad \psi(r)=r^{(s-1) / 2 s} \Psi(q) .
$$

The transformed equation is

$$
-\hbar^{2} \frac{d^{2} \Psi(q)}{d q^{2}}+Q(q) \Psi(q)=0
$$

where

$$
\begin{aligned}
Q(q) & =2 m s^{2} q^{2 s-2}\left(V\left(q^{s}\right)-E\right) \\
& +\frac{\hbar^{2}}{q^{2}}\left(s^{2}(l+1 / 2)^{2}-\frac{1}{4}\right) .
\end{aligned}
$$

R. E. Langer [12] has used the particular cases $s=2$ and $s=\infty$ (exponential substitution) when he applied the WKB approximation to the Kepler problem. In [9], the values $s=1$ for $l=0$ and $s=2$ for $l \neq 0$ were chosen in the framework of the new modified WKB method [8] in the case of the power-law potentials. In the present paper, $s$ is a variational parameter. The optimal value of this parameter will be found by means of minimizing the integral discrepancy in accordance with a new improved variational approach verified in [13].

\section{Modified WKB method}

The WKB approach deals with the logarithmic derivative $Y(q)=d \ln \Psi(q) / d q$. The WKB series are the asymptotic expansions in powers of Plank's constant $\hbar$ of two particular logarithmic derivatives $Y^{ \pm}(q)$. The nontrivial properties of the WKB series were investigated in $[14,15]$. The usual WKB approximations contain a finite number of leading terms $Y_{n}^{ \pm}(q)$ of the complete expansions. These approximations are not valid at the turning points, where $Q(q)=0$.

The analysis of the well-known structure of the leading $Y_{n}^{ \pm}(q)$ and recursion relations allows us to reconstruct the asymptotic WKB series as the infinite sums of new partial asymptotic series [8]. The complete series are approximated by a finite number of leading partial series. Explicit summations of two leading partial series [8] and their generalizations [9] give the new approximate logarithmic derivative

$$
Y_{a p p}(q) \equiv Y(q ; t)=b_{1}(q) y_{1}(a ; t)+b_{2}(q) y_{2}(a ; t)
$$

with a mixture parameter $t$. The notations introduced are

$$
\begin{gathered}
a(q)=\frac{1}{\hbar^{2 / 3}} \frac{Q(q)}{|d Q(q) / d q|^{2 / 3}}, \quad b_{1}(q)=\frac{1}{\hbar^{2 / 3}} \frac{d Q(q) / d q}{|d Q(q) / d q|^{2 / 3}} \\
b_{2}(q)=\frac{d^{2} Q(q) / d q^{2}}{d Q(q) / d q}
\end{gathered}
$$


The functions

$$
\begin{gathered}
y_{1}(a ; t)=\frac{d}{d a} \ln (\operatorname{Ai}(a)+t \operatorname{Bi}(a)) \\
y_{2}(a ; t)=\frac{1}{30}\left[-8 a^{2}\left(y_{1}(a ; t)\right)^{2}-3-4 a y_{1}(a ; t)+8 a^{3}\right]
\end{gathered}
$$

are expressed in terms of the well-studied Airy functions $\operatorname{Ai}(a)$ and $\operatorname{Bi}(a)[16]$.

It is not surprising that the asymptotics of our approximation coincide with the WKB asymptotics far away from the turning points. At the same time our approximation reproduces the known [17] satisfactory approximation near the turning points.

Now we can construct the approximate radial wave functions for the bound states.

At the origin $(r \rightarrow 0, q \rightarrow 0)$ there exist the exact expressions

$$
\begin{gathered}
\psi_{e x}(r) \rightarrow r^{l+1}, \quad \Psi_{e x}(q) \rightarrow q^{s l+(s+1) / 2}, \\
Y_{e x}(q) \rightarrow \frac{s l+(s+1) / 2}{q}
\end{gathered}
$$

First, we must reproduce the correct limiting behavior. This requirement determines the value of $t$ as

$$
t_{0}=\frac{-c(l, s) \operatorname{Ai}\left(a_{0}\right)+a_{0}\left(d \operatorname{Ai}\left(a_{0}\right) / d a_{0}\right)}{c(l, s) \operatorname{Bi}\left(a_{0}\right)-a_{0}\left(d \operatorname{Bi}\left(a_{0}\right) / d a_{0}\right)},
$$

where

$$
\begin{gathered}
c(l, s)=1-\sqrt{1+\frac{5}{4}\left(\frac{8 a_{0}^{3}-3}{10}+s(l+1 / 2)+1 / 2\right)} \\
a_{0}=\left(\frac{s^{2}}{4}(l+1 / 2)^{2}-\frac{1}{16}\right)^{1 / 3} .
\end{gathered}
$$

Two real turning points $q_{-}$and $q_{+}\left(Q\left(q_{ \pm}\right)=0\right)$ separate three regions.

In the first region, where $0<q<q_{-}$, we select the unique approximate particular logarithmic derivative $Y\left(q ; t_{0}\right)$. In the second region, where $q_{-}<q<q_{+}$, we must describe the oscillatory solution of the original Schrödinger equation (2). Therefore in this case we select two approximate particular logarithmic derivatives $Y(q ;+i)$ and $Y(q ;-i)$. In the third region, where $q>q_{+}$, we must describe only the decreasing solution of the original Schrödinger equation. Therefore in this case we select the approximate particular logarithmic derivative $Y(q ; 0)$ or $Y(q ; \infty)$ in accordance with the sign of $d Q(q) / d q$. Note that in the case where $l=0$ and $s=1$ we put $q_{-}=0$.

The turning points are ordinary nonsingular points in our approach in contrast with the conventional WKB method. Matching particular solutions at the turning points we obtain the continuous approximate radial wave function

$$
\psi_{a p p}(r, s)=\left\langle r \mid \psi_{a p p}(s)\right\rangle=N_{a p p} r^{(s-1) / 2 s} \Psi_{a p p}(q),
$$

where $\Psi_{a p p}(q)$ is represented as

$$
\Psi_{1}(q)=\cos \phi \exp \left(-\int_{q}^{q_{-}} Y\left(q^{\prime} ; t_{0}\right) d q^{\prime}\right)
$$

if $0<q<q_{-}$,

$$
\begin{aligned}
\Psi_{2}(q) & =\exp \left(\int_{q_{-}}^{q} \frac{Y\left(q^{\prime} ;+i\right)+Y\left(q^{\prime} ;-i\right)}{2} d q^{\prime}\right) \\
& \times \cos \left(\int_{q_{-}}^{q} \epsilon \frac{Y\left(q^{\prime} ;+i\right)-Y\left(q^{\prime} ;-i\right)}{2 i} d q^{\prime}-\phi\right)
\end{aligned}
$$

if $q_{-}<q<q_{+}$,

$$
\begin{aligned}
\Psi_{3}(q) & =\frac{(-1)^{n}}{2} \exp \left(\int_{q_{-}}^{q_{+}} \frac{Y\left(q^{\prime} ;+i\right)+Y\left(q^{\prime} ;-i\right)}{2} d q^{\prime}\right) \\
\times \exp \left(\int _ { q _ { + } } ^ { q } \left[\frac{Y\left(q^{\prime} ; \infty\right)+Y\left(q^{\prime} ; 0\right)}{2}\right.\right. & \\
& \left.\left.-\epsilon \frac{Y\left(q^{\prime} ; \infty\right)-Y\left(q^{\prime} ; 0\right)}{2}\right] d q^{\prime}\right)
\end{aligned}
$$

if $q>q_{+}$.

Here $\epsilon=(d Q(q) / d q)|d Q(q) / d q|^{-1}, \phi=\frac{\pi}{3}-\arctan t_{0}$, $q=r^{1 / s}$. The normalization constant $N_{a p p}$ is determined by the standard condition

$$
\left\langle\psi_{\text {app }}(s) \mid \psi_{\text {app }}(s)\right\rangle=1 .
$$

We have the new quantization condition

$$
\begin{gathered}
\int_{q_{-}}^{q_{+}} \epsilon \frac{Y(q ;+i)-Y(q ;-i)}{2 i} d q=\pi(n+1 / 3)+\phi \\
n=0,1,2 \ldots
\end{gathered}
$$

Note that up to now the value of $s$ is not fixed. We consider $s$ as a variational parameter and $\psi_{a p p}(r, s)$ as a trial function in the variational approach.

\section{Application to the Gaussian potential}

It is convenient to apply our approximation using the dimensionless quantities

$$
x=\frac{r}{R}, \quad e=\left(\frac{2 m R^{2}}{\hbar^{2}}\right) E .
$$

Then the Schrödinger equation is rendered to be

$$
\hat{H} \psi(x)-e \psi(x)=0
$$

with the Hamiltonian

$$
\hat{H}=-\frac{d^{2}}{d x^{2}}+V(x)+\frac{l(l+1)}{x^{2}},
$$

where the Gaussian potential

$$
V(x)=-A \exp \left(-x^{2}\right)
$$

quickly tends to zero from $-A$. This potential results in a finite number of energy levels.

In order to choose the optimal value of a variational parameter within the framework of the variational approach it is worthwhile to recast the Schrödinger equation in the form

$$
\frac{(\hat{H}+A) \psi(x)}{\langle\psi|\hat{H}+A| \psi\rangle}-\psi(x)=0
$$


Table 1. Properties of the proposed approximation for small $l$

\begin{tabular}{rccrrrr}
\hline$l$ & $n$ & $s_{o}$ & $d$ & vir & $-e_{\text {app }}$ & $-e_{\text {num }}$ \\
\hline 0 & 0 & 1 & $1.629 \times 10^{-4}$ & $1.787 \times 10^{-2}$ & 341.891 & 341.895 \\
& 1 & 1 & $3.329 \times 10^{-6}$ & $6.572 \times 10^{-3}$ & 269.644 & 269.644 \\
& 2 & 1 & $6.589 \times 10^{-7}$ & $4.663 \times 10^{-3}$ & 203.983 & 203.984 \\
& 3 & 1 & $2.577 \times 10^{-7}$ & $4.073 \times 10^{-3}$ & 145.378 & 145.378 \\
& 4 & 1 & $1.407 \times 10^{-7}$ & $3.928 \times 10^{-3}$ & 94.4575 & 94.4577 \\
& 5 & 1 & $1.001 \times 10^{-7}$ & $4.083 \times 10^{-3}$ & 52.1434 & 52.1436 \\
& 6 & 1 & $1.139 \times 10^{-7}$ & $4.708 \times 10^{-3}$ & 19.9660 & 19.9663 \\
& 7 & 1 & $3.591 \times 10^{-7}$ & $6.850 \times 10^{-3}$ & 1.34411 & 1.3473 \\
\hline 1 & 0 & 1.373 & $1.315 \times 10^{-2}$ & $6.751 \times 10^{-2}$ & 304.302 & 304.468 \\
& 1 & 2.480 & $1.414 \times 10^{-4}$ & $6.554 \times 10^{-3}$ & 235.441 & 235.450 \\
& 2 & 4.737 & $2.922 \times 10^{-5}$ & $-3.246 \times 10^{-3}$ & 173.242 & 173.244 \\
& 3 & 5.648 & $1.306 \times 10^{-5}$ & $-2.042 \times 10^{-3}$ & 118.384 & 118.384 \\
& 4 & 5.889 & $8.565 \times 10^{-6}$ & $-3.672 \times 10^{-4}$ & 71.6234 & 71.6236 \\
& 5 & 5.978 & $5.904 \times 10^{-6}$ & $1.125 \times 10^{-3}$ & 34.1295 & 34.1299 \\
& 6 & 5.957 & $3.347 \times 10^{-6}$ & $2.561 \times 10^{-3}$ & 8.08248 & 8.0833 \\
\hline
\end{tabular}

Table 2. Properties of the proposed approximation for large $l$

\begin{tabular}{rrrrrrr}
\hline$l$ & $n$ & $s_{o}$ & $d$ & vir & $-e_{\text {app }}$ & $-e_{\text {num }}$ \\
\hline 8 & 0 & 2.301 & $6.488 \times 10^{-4}$ & $2.316 \times 10^{-3}$ & 75.0905 & 75.2 \\
& 1 & 2.271 & $4.722 \times 10^{-6}$ & $2.682 \times 10^{-3}$ & 32.8301 & 32.8 \\
& 2 & 2.219 & $7.458 \times 10^{-7}$ & $4.729 \times 10^{-3}$ & 1.12976 & \\
\hline 9 & 0 & 2.123 & $4.668 \times 10^{-4}$ & $2.561 \times 10^{-3}$ & 47.7841 & 47.9 \\
& 1 & 2.746 & $3.646 \times 10^{-6}$ & $2.039 \times 10^{-3}$ & 10.4791 & 10.5 \\
\hline 10 & 0 & 3.089 & $3.268 \times 10^{-4}$ & $5.246 \times 10^{-4}$ & 22.1697 & \\
\hline
\end{tabular}

for the normalized eigenfunctions $\psi(x)=\langle x \mid \psi\rangle$. In accordance with (21) a discrepancy vector is introduced:

$$
|D(s)\rangle=\frac{(\hat{H}+A)\left|\psi_{a p p}(s)\right\rangle}{\left\langle\psi_{a p p}(s)|\hat{H}+A| \psi_{a p p}(s)\right\rangle}-\left|\psi_{a p p}(s)\right\rangle
$$

for a normalized trial function $\psi_{a p p}(x, s)=\left\langle x \mid \psi_{a p p}(s)\right\rangle$ which is not an exact solution of the Schrödinger equation. Following [13], an integral discrepancy can be determined:

$$
d(s)=\langle D(s) \mid D(s)\rangle=\frac{\left\langle\psi_{a p p}(s)\left|(\hat{H}+A)^{2}\right| \psi_{a p p}(s)\right\rangle}{\left\langle\psi_{a p p}(s)|\hat{H}+A| \psi_{a p p}(s)\right\rangle^{2}}-1 .
$$

This discrepancy characterizes goodness of the approximation and is equal to zero for an exact solution of the Schrödinger equation.

We propose to determine the optimal value $s_{o}$ of a variational parameter from the minimality condition for the integral discrepancy. Note that we look for the absolute minimum of $d(s)$.

In addition to the integral discrepancy we use a relative virial error

$$
\operatorname{vir}(s)=\frac{\left\langle\psi_{\text {app }}(s)\left|-\frac{d^{2}}{d x^{2}}+\frac{l(l+1)}{x^{2}}\right| \psi_{a p p}(s)\right\rangle}{\left\langle\psi_{a p p}(s)\left|\frac{1}{2} x \frac{d V(x)}{d x}\right| \psi_{a p p}(s)\right\rangle}-1
$$

in order to estimate quality of the proposed approximation. The value of $\operatorname{vir}(s)$ is equal to zero for the exact solutions.

At last we present our predictions concerning the energy values

$$
e_{a p p}(s)=\left\langle\psi_{a p p}(s)|\hat{H}| \psi_{a p p}(s)\right\rangle
$$

which are calculated in accordance with the variational approach with the help of the proposed approximate wave functions $\psi_{\text {app }}(x, s)$.

Following previous papers [3-7], we give the numerical results for the particular value $A=400$. In this case the bound states exist for $l=0-10$. Full number of bound states is 51 . We show our results for small $l$ in Table 1 and for large $l$ in Table 2 .

In Tables 1,2 we estimate our approximation when the value of a variational parameter is optimal $\left(s=s_{o}\right)$ and hence the integral discrepancy is minimal. We see that $s_{o}=1$ for $l=0$ and various $n$ as in [9] but the value of $s_{o}$ depends on $n$ if $l \neq 0$. Here we use the notations $d=d\left(s_{o}\right), \operatorname{vir}=\operatorname{vir}\left(s_{o}\right)$ and $e_{a p p}=e_{a p p}\left(s_{o}\right)$. Our approximate energy levels $e_{a p p}$ are compared with the results $e_{\text {num }}$ of the precise numerical calculations [5] for small $l$. Note that the accuracy of our approximation is fairly good for $l=3-7$ and values of $e_{a p p}$ are close to precise values of $e_{\text {num }}[4,5]$. There are only unpublished numerical results of Buck quoted in [3] in the case of large $l$. One can see that two states $l=8, n=3$ and $l=10, n=0$ were omitted in [3]. We perform a more detail description of these states.

Figures 1,2 demonstrate continuity of the proposed normalized approximate radial wave functions $\psi_{\text {app }}(x)=\psi_{\text {app }}\left(x, s_{o}\right)$ and their first derivatives $\psi_{\text {app }}^{\prime}(x)=d \psi_{\text {app }}(x) / d x$. Here solid lines correspond to the state $l=8, n=3$ and dashed lines correspond to the state $l=10, n=0$. The behavior of the first derivatives shows that the second derivatives are continuous too. 


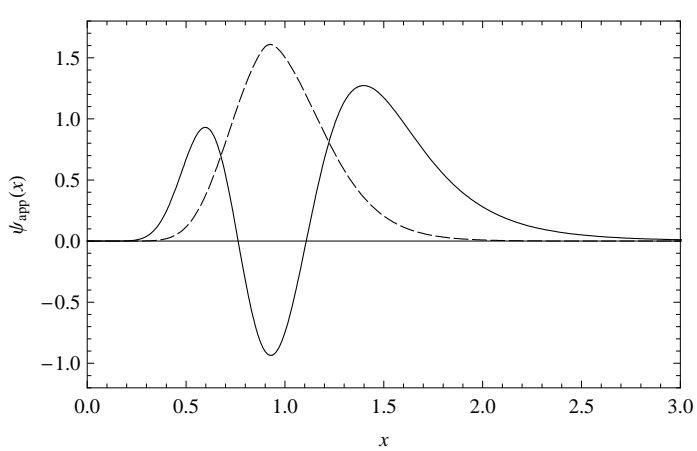

Figure 1. Radial wave functions.

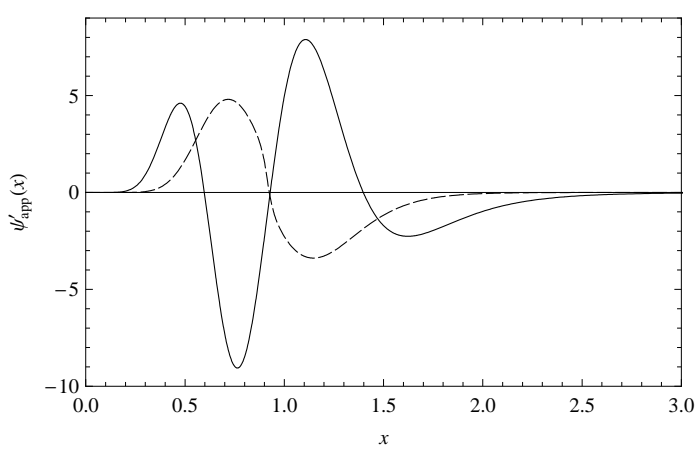

Figure 2. Derivatives of radial wave functions.

\section{Conclusion}

The modified WKB method yields the satisfactory qualitative description of the wave functions in the case of the radial Schrödinger equation. The application to the Gaussian potential demonstrates the sufficiently well quantitative properties of the proposed approach. In addition, the use of new method allows us to predict the existence of bound states which have been missed earlier.

\section{REFERENCES}

[1] B. Buck, H. Friedrich, C. Wheatley. Local potential models for the scattering of complex nuclei, Nucl. Phys. A, Vol.275, No.1, 246-268, 1977.

[2] S. S. Gomez, R. H. Romero. Few-electron semiconductor quantum dots with Gaussian confinement, Cent. Eur. J. Phys., Vol.7, No.1, 12-20, 2009.

[3] G. Stephenson. Eigenvalues of the Schrödinger equation with a Gaussian potential, J. Phys. A: Math. Gen., Vol.10, No.12, L229-L232, 1977.
[4] C. S. Lai. On the Schrödinger equation for the gaussian potential $-A \exp \left(-\lambda r^{2}\right)$, J. Phys. A: Math. Gen., Vol.16, No.6, L181-L185, 1983.

[5] R. E. Crandall. Fast eigenvalue algorithm for central potentials, J. Phys. A: Math. Gen., Vol.16, No.12, L395L399, 1983.

[6] A. Chatterjee. 1/N expansion for Gaussian potential, J. Phys. A: Math. Gen., Vol.18, No.12, 2403-2408, 1985.

[7] K. Köksal. A simple analytical expression for bound state energies for an attractive Gaussian confining potential, Phys. Scr., Vol.86, No.3, 035006, 2012.

[8] V. V. Kudryashov, Yu. V. Vanne. Explicit summation of the constituent WKB series and new approximate wave functions, J. Appl. Math., Vol.2, No.6, 265-275, 2002.

[9] V. V. Kudryashov. New approximate radial wave functions for power-law potentials, Nonlinear Phenomena in Complex Systems, Vol.9, No.4, 403-409, 2006.

[10] J. Heading. An Introduction to the Phase-Integral Methods, Willey, New York, 1962.

[11] N. Fröman, P. O. Fröman. Phase-Integral Method: Allowing Nearlying Transition Points, Springer-Verlag, New York, 1996.

[12] R. E. Langer. On the connection formulas and the solutions of the wave equation, Phys. Rev., Vol.51, No.8, 669-676, 1937.

[13] V. V. Kudryashov, V. I. Reshetnyak. Improved perturbative-variational approach for excited states, Nonlinear Phenomena in Complex Systems, Vol.11, No.3, 385-389, 2008.

[14] C. M. Bender, K. Olaussen, P. S. Wang. Numerological analysis of the WKB approximation in large order, Phys. Rev. D, Vol.16, No.6, 1740-1748, 1977.

[15] M. Robnik, V.G. Romanovski. Some properties of WKB series, J. Phys. A: Math. Gen., Vol.33, No.28, 50935104, 2000.

[16] M. Abramovitz, I. A. Stegun (eds.). Handbook of Mathematical Functions, Dover Publications, New York, 1970.

[17] C. M. Bender, S. A. Orszag. Advanced Mathematical Methods for Scientists and Engineers, McGraw-Hill, New York, 1978. 